\title{
Bronchial Fibroepithelial Polyp: A Rare and Challenging Diagnosis
}

\author{
Francisco Barbosa De Araújo Neto ${ }^{*}$, Ana Flávia Pina Ferreira1, \\ Ellen Caroline Toledo Do Nascimento ${ }^{2}$, Ricardo Francisco Cintra Zagatti' \\ Mark Wanderley ${ }^{1}$, Thiana Pozzatto Rogrigues', Tiago Castello Branco Lyra ${ }^{3}$, \\ Vinícius Martins Valois ${ }^{3}$, Márcio Valente Yamada Sawamura ${ }^{1}$
}

${ }^{1}$ Department of Radiology, The Medical School, The University of São Paulo, São Paulo, Brazil ${ }^{2}$ Department of Pathology, The Medical School, The University of São Paulo, São Paulo, Brazil ${ }^{3}$ Department of Radiology, Hospital Heliópolis, São Paulo, Brazil Email: ^Bilbanmaster@gmail.com, Flavinhaferreira@gmail.com, Ellentn@uol.com.br, Ricardofczabc@gmail.com, Mark.wanderley@gmail.com, Thianapozzatto.tr@gmail.com, Tiagocbl@hotmail.com,

Viniciusmvalois@gmail.com, Marcio.sawamura@hc.fm.usp.br

How to cite this paper: De Araújo Neto, F.B., Ferreira, A.F.P., Do Nascimento, E.C.T., Zagatti, R.F.C., Wanderley, M., Rogrigues, T.P., Lyra, T.C.B., Valois, V.M. and Sawamura, M.V.Y. (2017) Bronchial Fibroepithelial Polyp: A Rare and Challenging Diagnosis. Open Journal of Medical Imaging, 7, 163-168.

https://doi.org/10.4236/ojmi.2017.74016

Received: September 25, 2017

Accepted: October 15, 2017

Published: October 18, 2017

Copyright $\odot 2017$ by authors and Scientific Research Publishing Inc. This work is licensed under the Creative Commons Attribution International License (CC BY 4.0).

http://creativecommons.org/licenses/by/4.0/

\begin{abstract}
Context: Bronchial fibroepithelial polyp is a very rare benign endobronchial tumor. Typically, it is a slow growing lesion, and the differential diagnosis includes other benign and malignant endobronchial tumors. Case Report: We report a case of a 75-year-old female who complained of intermittent productive cough. Computed tomography of the thorax showed a solid nodule with lobulated contours in the right main bronchus and subtotal atelectasis of the right lower lobe. Bronchoscopy with biopsy confirmed the diagnosis of a bronchial fibroepithelial polyp. Conclusion: This case is illustrative and demonstrates the importance of imaging methods and of histopathological correlation for the accurate diagnosis of this benign treatable lesion.
\end{abstract}

\section{Keywords}

Tomography, Bronchoscopy, Bronchial Diseases, Polyps

\section{Introduction}

Fibroepithelial polyps are a common type of tumor in the skin or genitourinary tract. However, Bronchial fibroepithelial polyps represent a rare type of benign pseudoneoplastic lesion with endobronchial growth. Making a proper diagnosis and selecting the appropriate treatment of such polyps is rather difficult once they can mimic a malign neoplasm. At bronchoscopy, bronchial fibroepithelial polyps are reported to appear as rounded, whitish polypoid lesions with a firm 
consistency and a glistening, fleshy, smooth surface [1] [2].

Histologically, these lesions are composed of collagen fibers in a stroma covered by normal respiratory epithelium [3]. In symptomatic patients, the lesion should be entirely removed, although no consensus has been reached in literature concerning the most correct approach. Because recurrence is uncommon, a complete excision of small lesions with multiple bronchoscopic biopsies may be sufficient [4].

We will report a case of a bronchial fibroepithelial polyp. Although rare, this is a differential diagnosis of endobronchial pathologies.

\section{Case Report}

Female patient, 75-year-old, who complained of intermittent productive cough with hyaline expectoration. The patient underwent thoracic computed tomography, that showed a solid small nodule with lobulated contours, located in the right main bronchus (Figure 2 and Figure 3). The lesion partially obstructed the bronchus and caused subtotal atelectasis of right lung's lower lobe (Figure 4). The patient underwent bronchoscopy with biopsy that confirmed the bronchial fibroepithelial polyp (Figures 1-5).

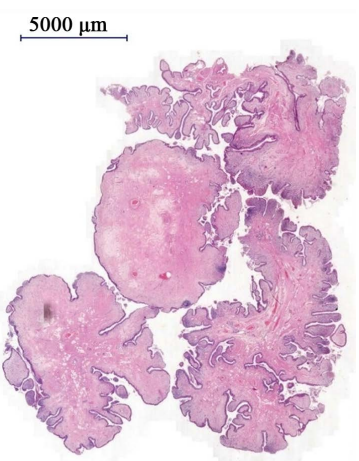

(a)

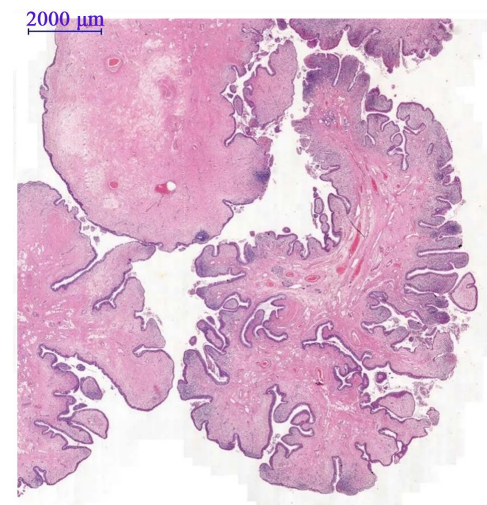

(b)

Figure 1. Histological sections showed a polyp covered by a normal respiratory epithelium and fibrovascular stroma with mild chronic inflammatory infiltrate composed of lymphocytes and plasma cells ((a) Hematoxylin and Eosin stain, magnification $\times 5$; (b) $\mathrm{H}$ $\&$ E stain, magnification $\times 10)$.

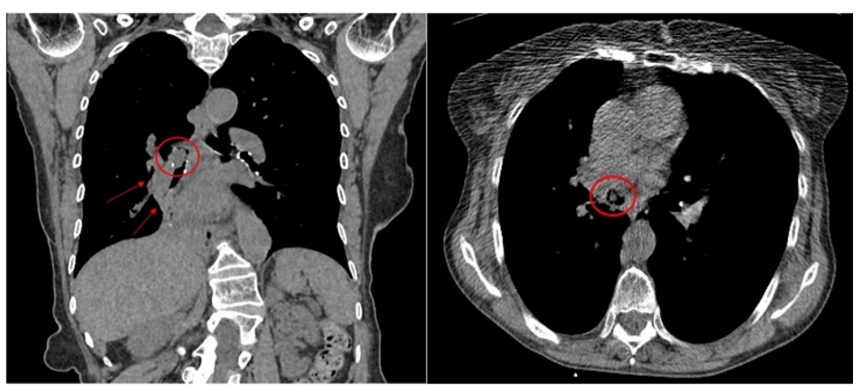

Figure 2. Sagittal reformatted and axial mediastinal window CT images shows lobulated endobronchial solid nodule partially obstructing the right main bronchus (red circle). 


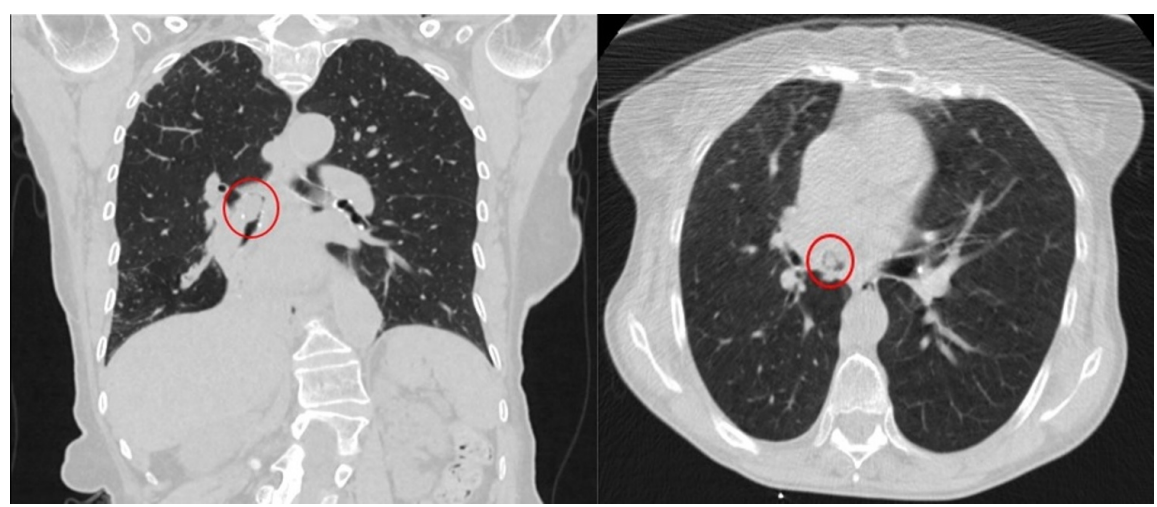

Figure 3. Sagittal reformatted and axial lung window CT images shows lobulated endobronchial solid nodule partially obstructing the right main bronchus (red circle).

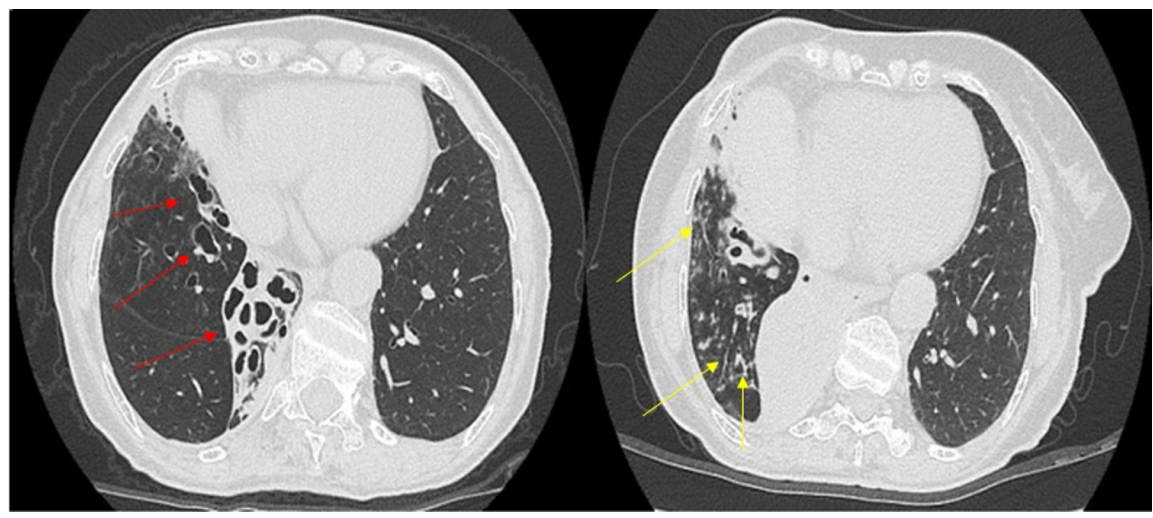

Figure 4. Axial lung window CT images show bronchiectasis (red arrows) and fibroatelectasis (yellow arrows) due to the obstructive effect of the endobronchial lesion.

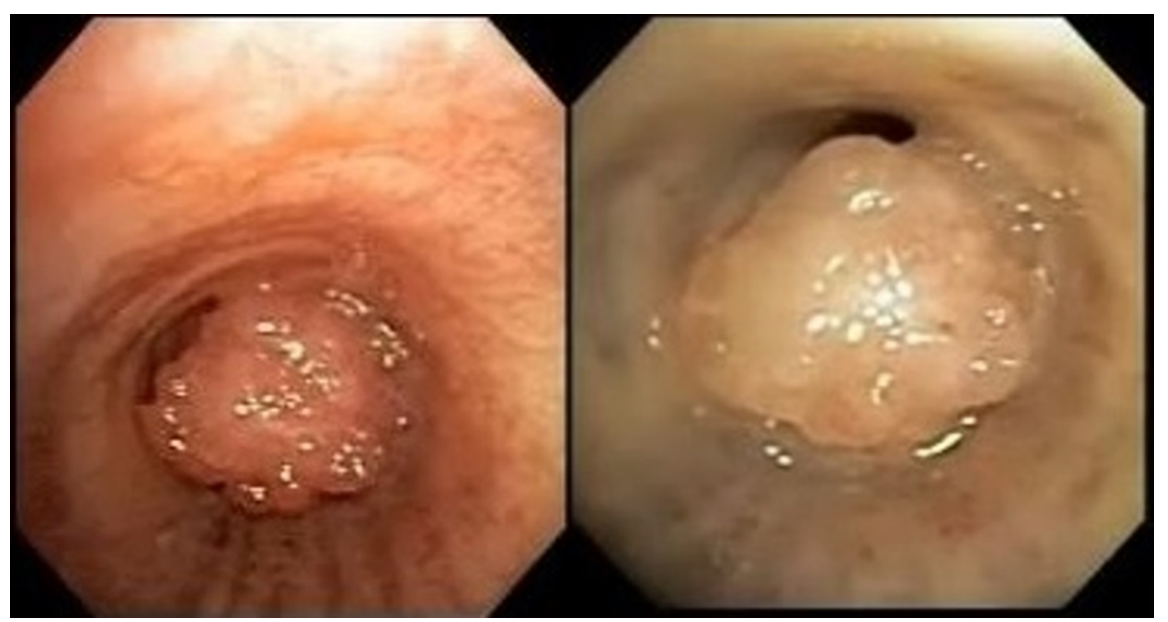

Figure 5. Bronchoscopy images demonstrate a shiny polypoid lesion with lobulated contours that partially obstructed the right main bronchus.

The lesion was ressected by bronchoscopy and the patient is being followed for 3 years. Since the ressection, the patient had two episodes of pneumonia and intermittent cough with hyaline secretion. 


\section{Discussion}

Although benign endobronchial polyps were first described in 1930 by Patterson, who termed them as neoplasms, it is rarely reported in the literature [5]. Inflammatory polyps in the respiratory system are defined as tumor-like non-neoplastic endobronchial lesions according to the WHO classification which was published in 1999 [5].

Fibroepithelial polyps of the bronchi are rare with only a few case reports in the English literature [6] [7]. According to these case reports, it manifests as an endobronchial polypoid nodule that is covered with normal respiratory mucosa, consisting of fibrovascular stroma with or without few inflammatory cells and adipocytic components [6] [7].

The etiology of fibroepithelial polyps of the bronchus is unclear. Some reports have classified fibroepithelial polyps as inflammatory, while others have argued that the inflammation is a result of the intermittent respiratory tract obstruction caused by its mass effect [7]. Other studies show that the pathogenesis of this lesion is unknown, although chronic bronchial stimulation by several agents (e.g. smoke, infections, aspiration) has been speculated as a possible cause, and bronchial fibroepithelial polyp probably represents a reactive, pseudoneoplastic growth rather than a true tumor [2] [8].

The histologic examination revealed a polyp covered by normal respiratory epithelium and fibrovascular stroma with mild chronic inflammatory infiltrate composed of lymphocytes and plasma cells (Figure 1). Several chronic inflammatory etiologic factors such as foreign body aspiration, prolonged mechanical ventilation, asthma, chronic sinusitis, chronic smoke inhalation, and mycobacterial infections may potentially be the cause [5].

The presumptive diagnosis of this lesion is made by radiological methods such as chest X-ray and computed tomography of the chest. In our case and corroborating the reports of other cases, after visualization of the lesion by computed tomography, we demonstrated that the lesion presents peculiar characteristics such as lobulated and/or microlobulated contours, endobronchial localization, with solid texture and expansive aspect, discretely touching the adjacent bronchial walls (Figure 2 and Figure 3). We also found pulmonary alterations secondary to the obstructive effect of the lesion, such as accumulation of mucous secretion in the underlying bronchi, atelectasis of lung segments and lobes, and changes associated with inflammatory and infectious processes due to mucoid impaction and reactivity (Figure 4).

Differential diagnosis includes malignant lesions such as carcinoid tumors, metastasis, adenoid cystic carcinoma, mucoepidermoid carcinoma and benign lesions such as foreign body aspiration, endobronchial lipoma and hamartoma [9].

After the diagnosis of the lesion by tomography, it is recommended to perform a bronchoscopy with biopsy to characterize the lesion and to collect material for histopathological evaluation. In our case, a shiny polypoid lesion with lobulated contours that partially obstructed the right main bronchus was cha- 
racterized (Figure 5). Bronchoscopy appearance of fibroepithelial polyp is suggestive of the diagnosis when dealing with a whitish and glistening, firm, polypoid lesion with rounded or lobulated borders, as previously reported [8].

The definitive and curative treatment of the lesion is performed by surgical or bronchoscopy resection, indicated to avoid post obstructive complications as well for confirmation of the diagnosis [2] [8]. In some instances, fibroepithelial polyps may be multiple or even recur but the clinical course is usually favorable [2].

This case is illustrative and important to be shared with radiologists and the medical community, since it is a rare benign lesion with a potential endoscopic treatment, that could be misinterpreted as a malignant lesion. Also, the case emphasizes the importance of computed tomography for evaluation of airway lesions, elevating the accuracy for this challenging diagnosis.

\section{Conclusion}

Although rare, fibroepithelial polyps should be included in the differential diagnosis of endobronchial tumors. Radiologists, pulmonologists and the medical community should be aware of this benign tumor, imaging workup and management.

\section{Funding}

No funding was used in this work.

\section{Conflict}

There is no conflict of interest of the authors in this work/document.

\section{References}

[1] Casalini, E., Cavazza, A., Andreani, A., et al. (2017) Bronchial Fibroepithelial Polyp: A Clinico-Radiologic, Bronchoscopic, Histopathological and In-Situ Hybridisation Study of 15 Cases of a Poorly Recognised Lesion. The Clinical Respiratory Journal, 11, 43-48. https://doi.org/10.1111/crj.12300

[2] Li, M., Zhang, G., Peng, A., et al. (2013) Bronchial Fibroepithelial Polyp: A Case Report and Review of the Literature. Internal Medicine, 52, 373-376. https://doi.org/10.2169/internalmedicine.52.8864

[3] Ushiki, A., Yasuo, M., Tanabe, T., et al. (2008) A Rare Case of a Tracheal Fibroepithelial Polyp Treated by an Endobronchial Resection. Internal Medicine, 47, 1723-1726. https://doi.org/10.2169/internalmedicine.47.1241

[4] Maskey, A.P., Almeida, F. and Farver, C. (2012) Endobronchial Fibroepithelial Polyp. Journal of Bronchology \& Interventional Pulmonology, 19, 313-314. https://doi.org/10.1097/LBR.0b013e31826c5f42

[5] Schnader, J., Harrell, J., Mathur, P., et al. (2002) Bronchiectasis and Endobronchial polyps. Chest, 121, 637-643. https://doi.org/10.1378/chest.121.2.637

[6] Dinçer, I., Demir, A., Akin, H., et al. (2005) A Giant Endobronchial Inflammatory Polyp. The Annals of Thoracic Surgery, 80, 2353-2356.

https://doi.org/10.1016/j.athoracsur.2004.07.038 
[7] Amin, P.B. and Baciewicz, F. (2009) Benign Fibroepithelial Polyp Arising in the Bronchus: A Case Report and Review of the Literature. Archives of Surgery, 144, 1081-1083. https://doi.org/10.1001/archsurg.2009.198

[8] Kang, H., Kim, T.S., Han, J., et al. (2012) Fibroepithelial Polyp of the Bronchus: CT and Histopathologic Findings. Korean Journal of Radiology, 13, 355-357. https://doi.org/10.3348/kjr.2012.13.3.355

[9] Webb, W.R. and Higgins, C.B. (2011) Thoracic Imaging: Pulmonary and Cardiovascular Radiology. 2nd Ed., Lippincott Williams \& Wilkins, Philadelphia, PA. 